\title{
Parameters of the neural network model
}

In this section, we presented the detailed parameters of the neural network model we used.

1) number of epochs: 500

2) learning rate: 0.3

3) momentum: 0.2

4) squash function: sigmoid function

5) loss function: least square function

6) initial weights: random value range from -0.05 to 0.05 